\title{
A Case Series Assessing the Effects of Lomitapide on Carotid Intima-Media Thickness in Adult Patients with Homozygous Familial Hypercholesterolaemia in a Real-World Setting
}

\author{
Dirk J. Blom (1) - Daniel Gaudet - Robert A. Hegele · Dharmesh S. Patel · \\ Jaimini Cegla · Genovefa Kolovou · Luis Masana Marin
}

Received: October 27, 2021 / Accepted: December 10, 2021 / Published online: February 23, 2022

(C) The Author(s) 2022

\begin{abstract}
Introduction: Homozygous familial hypercholesterolaemia (HoFH) is characterised by extremely elevated levels of low-density lipoprotein cholesterol (LDL-C) and results from multiple
\end{abstract}

Supplementary Information The online version contains supplementary material available at https:// doi.org/10.1007/s12325-021-02031-y.

\section{J. Blom ( $₫)$}

Department of Medicine, Division of Lipidology and Hatter Institute for Cardiovascular Research in Africa, University of Cape Town, Anzio Road Observatory, Cape Town 7925, South Africa e-mail: dirk.blom@uct.ac.za

\section{Gaudet}

Department of Medicine, University of Montreal, Montreal, Canada

R. A. Hegele

Department of Medicine, Western University, London, ON, Canada

D. S. Patel

Stern Cardiovascular Foundation, Memphis, USA

J. Cegla

Imperial Division of Diabetes, Endocrinology and

Metabolism, Imperial College London, London, UK

G. Kolovou

Lipid Center and Lipoprotein Apheresis Unit,

Metropolitan Hospital, Piraeus, Greece

L. M. Marin

Universitat Rovira I Virgili, IISPV, CIBERDEM, Reus, Spain mutations in genes affecting the LDL receptor pathway. Patients are at risk of premature atherosclerotic cardiovascular disease (ASCVD) and premature death. Lomitapide is a microsomal triglyceride transfer protein inhibitor developed to treat $\mathrm{HoFH}$, but cardiovascular outcome data are lacking.

Methods: We evaluated detailed data from five HoFH patients and one patient with heterozygous $\mathrm{FH}(\mathrm{HeFH})$ and a very severe phenotype. We also analysed confirmatory data from a further $8 \mathrm{HoFH}$ cases. In total, we analysed data from patients in seven global centres in six countries who were all treated with lomitapide with long-term follow-up. Carotid intima-media thickness (CIMT) imaging was recorded on an ad hoc basis to monitor ASCVD in HoFH.

Results: Lomitapide resulted in marked decreases in LDL-C of 56.8-93.9\% [77.7-93.9\% in the 6 initial cases (mean nadir $64.8 \pm 30.1 \mathrm{mg} / \mathrm{dL}) ; 56.8-86.0 \%$ in the 8 confirmatory cases (mean nadir $131.4 \pm 38.2 \mathrm{mg} /$ $\mathrm{dL}$ )]. CIMT regressed in $50 \%$ of cases (mean follow-up $5.0 \pm 3.1$ years in initial six cases, and $4.4 \pm 1.4$ years in confirmatory cases). In the remaining patients, CIMT showed little further change. In patients where assessments of plaque area were available, regression or stabilisation in CIMT was accompanied by clinically significant regression of plaque area.

Conclusions: Lomitapide reduces LDL-C levels in patients with HoFH and severe LDL-C phenotypes, and results in stabilisation and/or 
regression of CIMT, which is an established marker of ASCVD risk. Additional data are needed to determine if this confers a survival benefit in these very high-risk patients.

Keywords: Homozygous familial hypercholesterolemia; Lomitapide; Carotid intima-media thickness; Cardiovascular; Low-density lipoprotein cholesterol

\section{Key Summary Points}

The principal aim of lipid-lowering therapy in Homozygous familial hypercholesterolaemia (HoFH) is to avert or delay the onset of atherosclerotic cardiovascular disease (ASCVD).

The effect of lomitapide on markers of subclinical atherosclerosis has not been studied in clinical trials

The present case series examines changes in carotid intima-media thickness (CIMT) in 14 patients receiving lomitapide in the normal course of care.

Lomitapide substantially reduced Lowdensity lipoprotein cholesterol (LDL-C) levels in the patients with HoFH and resulted in stabilisation and/or regression of CIMT

Lomitapide may have the potential to delay or avert ASCVD progression in patients with $\mathrm{HoFH}$

\section{INTRODUCTION}

Homozygous familial hypercholesterolaemia (HoFH) is a rare metabolic condition caused by bi-allelic genetic defects in the low-density lipoprotein (LDL) receptor (LDL-R) pathway that lead to impaired uptake of LDL particles by the liver. This results in severely elevated plasma levels of LDL cholesterol (LDL-C) and premature, progressive atherosclerotic cardiovascular disease (ASCVD) [1-3].
The underlying molecular abnormalities resulting in the HoFH phenotype vary between patients. Patients may have homozygous, compound heterozygous or double heterozygous mutations in several genes encoding proteins involved in the LDL-R pathway. At present, HoFH causative mutations have been identified in $L D L R$ (encoding LDL-R), $A P O B$ (encoding apolipoprotein [apo] B), PCSK9 (encoding proprotein convertase subtilisin/kexin type 9), and LDLRAP1 (which encodes LDL-R adaptor protein 1). Most patients with HoFH have bi-allelic LDLR mutations.

Patients with HoFH usually display extremely elevated levels of LDL-C, but there is marked variability in the severity of the phenotype. Most patients have physical stigmata of hypercholesterolaemia such as tendinous and cutaneous xanthoma and/or corneal arcus [1]. Although ASCVD is generalised in patients with HoFH, the most common clinical manifestations are coronary artery disease and supravalvular as well as valvular aortic stenosis [1]. If HoFH goes untreated, the average age for development of ASCVD is 12.5 years and mean survival is 18 years [4]. To delay or avoid the onset of major, adverse cardiovascular events in $\mathrm{HoFH}$, early diagnosis and effective therapy are critical. The core principle for the treatment of $\mathrm{HoFH}$ is to reduce LDL-C levels as much as possible to reduce cumulative LDL-C exposure [1].

The European Atherosclerosis Society has set LDL-C targets for patients with HoFH in line with the notion that ASCVD risk is high or very high in all patients with HoFH. These targets are $<100 \mathrm{mg} / \mathrm{dL} \quad(<2.5 \mathrm{mmol} / \mathrm{L})$ in adults and $<135 \mathrm{mg} / \mathrm{dL} \quad(<3.5 \mathrm{mmol} / \mathrm{L})$ in children or $<70 \mathrm{mg} / \mathrm{dL}(<1.8 \mathrm{mmol} / \mathrm{L})$ in adults with diagnosed clinical ASCVD [1, 4].

Patients with $\mathrm{HoFH}$ are generally prescribed high-dose statins and ezetimibe, but statins act mainly by increasing LDL-R expression. As such, this therapeutic approach is much less effective in patients with $\mathrm{HoFH}$ compared with patients with other forms of hypercholesterolaemia [1]. Lipoprotein apheresis can further reduce LDL-C, but, due to its intermittent nature, it is often not possible to achieve complete LDL-C control in this manner [1]. The difficulties of adequately 
controlling LDL-C in patients with $\mathrm{HoFH}$ are well illustrated by data from the TAUSSIG study. TAUSSIG was a large study of well-treated patients with severe heterozygous $\mathrm{FH}$ or HoFH [5]. Despite aggressive lipid-lowering therapy (approximately $90 \%$ of HoFH patients received high-dose statins, 90\% received ezetimibe and $32 \%$ were treated with apheresis), baseline LDL$\mathrm{C}$ in HoFH patients was still $329 \pm 137 \mathrm{mg} / \mathrm{dL}$. Addition of evolocumab resulted in further reductions in LDL-C at Week 216 of $75 \pm 125 \mathrm{mg} / \mathrm{dL}$ [5]. These data show that it remains difficult to achieve LDL-C targets in patients with $\mathrm{HoFH}$, even with application of modern pharmacotherapy.

Lomitapide is a microsomal triglyceride transfer protein (MTP) inhibitor, approved as an adjunct to diet and other lipid-lowering agents, with or without apheresis, to reduce LDL-C in adult patients with HoFH $[6,7]$. Unlike conventional lipid-lowering agents that exert their LDL-C-lowering effect through upregulation of LDL-R activity, lomitapide acts independently of the receptor by reducing synthesis and subsequent export of apo B-containing lipoproteins from the liver and small intestine [8]. Phase 3 data demonstrated a 50\% reduction in LDL-C levels from baseline after 26 weeks of treatment [9]. These results were maintained through the overall planned 78 weeks of the pivotal trial. Currently, lomitapide is not yet available in all countries and access remains difficult for many patients in countries where health care payers do not provide reimbursement for lomitapide.

Despite the correlation of ASCVD risk with cumulative LDL-C exposure, there is very little published information on the effects of lomitapide on stabilisation and/or regression of atherosclerotic plaques and ultimately cardiovascular outcomes. A modelling study of cardiovascular event rates has estimated that the annualised major adverse cardiovascular event (MACE) rate is $2.0 \%$ for $\mathrm{HoFH}$ patients receiving lomitapide versus $26.1 \%$ for matched controls [10]. Carotid intima-media thickness (CIMT) is an established, non-invasive imaging surrogate for detection of subclinical atherosclerosis [11-13]. An image from a typical CIMT echo is given in Fig. 1. We therefore gathered data from clinical cases where CIMT measurements were

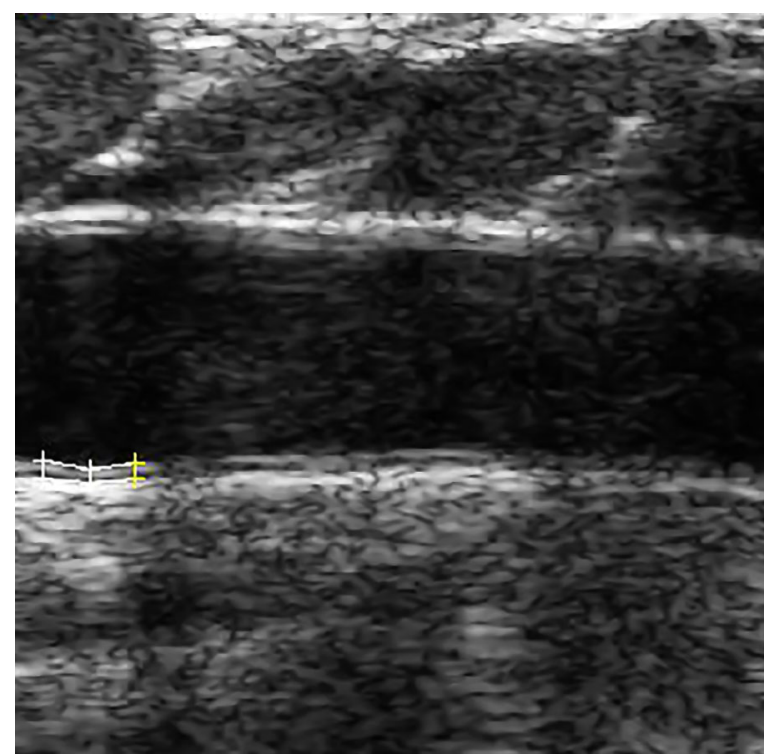

Fig. 1 Image from a typical, normal CIMT ultrasound test of the right common carotid artery. Central area shows the arterial lumen; crosses (lower left) indicate computerised measurement of CIMT. CIMT carotid intima-media thickness

available in HoFH patients receiving lomitapide with the aim of providing preliminary data on the effects of lomitapide on vascular health.

\section{CASE PRESENTATION}

Seven physicians with extensive experience in the treatment of $\mathrm{HoFH}$ provided data from patients treated in the course of normal clinical practice. Patient locations were South Africa, USA, UK, Spain $(n=1$ for all), Canada $(n=2)$ and Greece $(n=8)$. In almost all cases, and in accordance with the product label, lomitapide was commenced at a low dose (5 $\mathrm{mg} /$ day), and gradually escalated. Background lipid-lowering therapy (LLT) was provided according to local practices. In accordance with the lomitapide label, dietary counselling, vitamin $\mathrm{E}$ and essential fatty acid supplements were provided. All patients were counselled to observe a diet in which less than $20 \%$ of total daily energy was derived from fat. All patients underwent regular 
monitoring of liver function, along with other standard laboratory tests.

CIMT was measured according to local practices, and values mostly expressed as a mean of bilateral determinations. Ultrasound equipment and measurement techniques, as well as measurement intervals, differed between patients, which precluded a formal statistical analysis. Therefore, data are presented for individual patients. Baseline LDL-C was recorded at the clinic visit immediately prior to the commencement of lomitapide. LDL-C nadir values were defined as the lowest level achieved after lomitapide was commenced. The Greek patients are presented as a cohort analysis, validating the data from the first six identified cases.

This case series was conducted as a retrospective study of normal patient care and is not subject to Institutional Review Board approval. The study was performed in accordance with the Helsinki Declaration of 1964, and its later amendments. All the patients provided written consent for their details to be published in the current case series.

\section{RESULTS}

\section{Patient 1}

Patient 1 was a man with compound heterozygous p.Asp227Glu (defective) and p.Trp666Ter (null) mutations in $L D L R$. The patient had clinical evidence of atherosclerotic plaques (femoral and aortic vascular bruits) and supravalvular aortic stenosis at age 33 years when he first commenced lomitapide. He had not required surgery for these conditions. At the time lomitapide was commenced, the patient's LDL-C level was $385.5 \mathrm{mg} / \mathrm{dL}$-historical values were not available. The patient was receiving atorvastatin $80 \mathrm{mg} /$ day and ezetimibe $10 \mathrm{mg} /$ day. Lipoprotein apheresis (LA) was conducted every 2 weeks. At this time, carotid ultrasound revealed mean CIMT of $1.18 \mathrm{~mm}$ (Supplemental Fig. 1). Lomitapide was commenced at $5 \mathrm{mg} /$ day and escalated over the next 6 months to $40 \mathrm{mg} /$ day. LDL-C levels responded well and were brought down to as low as $80 \mathrm{mg} /$ dL (nadir). Three years after starting lomitapide,
LA was stopped, and the patient was maintained on lomitapide $40 \mathrm{mg} / \mathrm{day}$, with the background LLT unchanged. Liver enzymes remained normal throughout. In 2015, 7 years after lomitapide was commenced, a second carotid ultrasound was performed, and modest regression in CIMT to $1.11 \mathrm{~mm}$ was noted (Supplemental Fig. 1). LDL-C was maintained at approximately $100-180 \mathrm{mg} / \mathrm{dL}$, with no signs of cardiovascular disease progression. However, approximately 5 years after starting lomitapide, this patient underwent aortic valve and root replacement for supravalvular aortic stenosis. The patient developed fungal infective endocarditis post-surgery, and died following repeat surgery.

\section{Patient 2}

Patient 2 is a woman with a homozygous p.Trp87Arg (defective) mutation in the $L D L R$, conferring diminished LDL-R function. At diagnosis (age 14 years), and without LLT, the patient's LDL-C level was $823.7 \mathrm{mg} / \mathrm{dL}$ and characteristic xanthomas were evident. The patient had early onset coronary artery disease and had undergone angioplasty with stenting at age 37 . At age 41 years, the patient was receiving atorvastatin $80 \mathrm{mg} / \mathrm{day}$, ezetimibe $10 \mathrm{mg}$ /day and aspirin $80 \mathrm{mg} /$ day, but was not receiving LA. LDL-C levels remained high at $580 \mathrm{mg} / \mathrm{dL}$. At this point, lomitapide was commenced at $5 \mathrm{mg} /$ day and escalated to $20 \mathrm{mg}$ /day (Fig. 2). Twelve months later, LDL-C levels had reduced to approximately $200 \mathrm{mg} / \mathrm{dL}$. That month, carotid ultrasonography revealed mean CIMT of $0.82 \mathrm{~mm}$ and total plaque area of $147.1 \mathrm{~mm}^{2}$. Over the next 2 years, LDL-C levels gradually reduced to below $100 \mathrm{mg} / \mathrm{dL}$ with a nadir of just $35.2 \mathrm{mg} / \mathrm{dL}$. Carotid ultrasound revealed some regression to CIMT $0.65 \mathrm{~mm}$ (Fig. 2) and total plaque area $120.5 \mathrm{~mm}^{2}$. A coronary artery bypass graft procedure was performed in the same year, and the patient continues to be monitored closely for cardiac progression. 


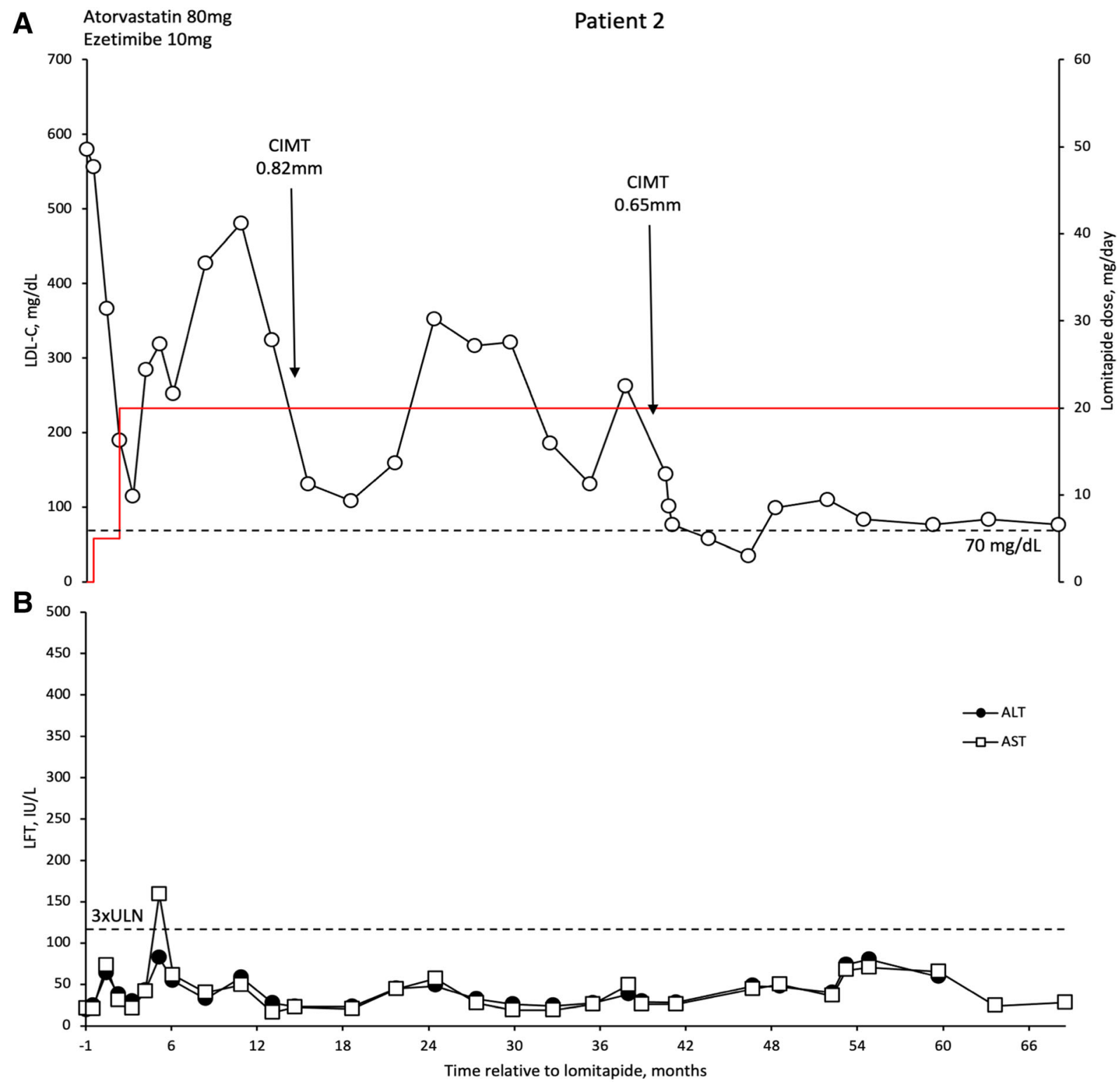

Fig. 2 A LDL-C levels, lomitapide dose (red line), CIMT, and B LFTs in Patient 2. ALT alanine aminotransferase, $A S T$ aspartate aminotransferase, CIMT carotid intimamedia thickness, $L A$ lipoprotein apheresis, $L D L-C$ low-

\section{Patient 3}

Patient 3 is a man with compound heterozygous null mutations; specifically, p.Gln739Ter (null) and an exon 1-6 copy number variation (null deletion). He was diagnosed at age 5 years, when biweekly plasmapheresis was initiated. At the age of 14 , his pre-apheresis LDL-C levels density lipoprotein cholesterol, $L F T$ liver function test, $U L N$ upper limit of normal

were $429.2 \mathrm{mg} / \mathrm{dL}$, and rosuvastatin $40 \mathrm{mg} /$ day, ezetimibe $10 \mathrm{mg} /$ day and aspirin $81 \mathrm{mg} /$ day were added. He entered the open-label phase 3 trial of lomitapide, reaching a final dose of $60 \mathrm{mg} /$ day after titration per study protocol (Supplemental Fig. 2). At that time, carotid ultrasound revealed mean CIMT of $0.76 \mathrm{~mm}$. On the 60-mg dose, LDL-C levels dropped to a 
nadir of $95.9 \mathrm{mg} / \mathrm{dL}$ and were maintained in the range of $150-430 \mathrm{mg} / \mathrm{dL}$ for the next 6 years, with variations related to periods of non-adherence and dietary indiscretion. During this period, there was evidence of regression in CIMT, with values gradually falling to $0.66 \mathrm{~mm}$. Unfortunately, the patient also experienced some elevation of liver function tests (LFTs) to $>3$-times the upper limit of normal (ULN), with the greatest peak in AST at $236 \mathrm{IU}$. Importantly, lomitapide was gradually down titrated to $40 \mathrm{mg} /$ day and then $20 \mathrm{mg} /$ day, and LA was reinstated to once every 2 weeks. LFTs normalised and the LDL-C was maintained in the same range as before. The last carotid ultrasound showed CIMT to be stable at $0.80 \mathrm{~mm}$.

\section{Patient 4}

Patient 4 is a man with a heterozygous p.Glu228Ter (null) mutation in LDLR and a high polygenic risk score of 16 out of 20 , which is in the $99^{\text {th }}$ percentile. LDL-C levels at diagnosis were approximately $350 \mathrm{mg} / \mathrm{dL}$, which is commensurate with a severe heterozygous phenotype. The patient was diagnosed with coronary artery disease and underwent initial coronary artery stenting at age 33 years with a history of cigarette smoking. He presented at age 40 years with unstable angina and underwent stenting of the left circumflex and left anterior descending artery. At this stage, the patient's lipid-lowering therapy was increased from simvastatin $40 \mathrm{mg}$ /day to atorvastatin $80 \mathrm{mg} /$ day.

At a follow-up visit when the patient was 40 years old, his LDL-C was $312 \mathrm{mg} / \mathrm{dL}$. The patient was advised to stop smoking and ezetimibe $10 \mathrm{mg} /$ day was added to existing atorvastatin $40 \mathrm{mg} /$ day. A clinical decision was made to commence off-label lomitapide at $5 \mathrm{mg} /$ day. LDL-C levels rapidly dropped to 128-136 mg/dL (Supplemental Fig. 3). Lomitapide dose was escalated to $30 \mathrm{mg} /$ day, and then adjusted to $20 \mathrm{mg} /$ day. A brief excursion in LFTs $>3$-times ULN required a brief down titration to $10 \mathrm{mg} /$ day. Commercial availability then allowed subcutaneous evolocumab $420 \mathrm{mg}$ monthly to be commenced with LDL-C levels persistently above $70 \mathrm{mg} / \mathrm{dL}$. Three years after commencing lomitapide, LDL-C levels reached a nadir of $33 \mathrm{mg} / \mathrm{dL}$. Lomitapide therapy was ceased in January 2017 due to gastrointestinal disturbances, and LDL-C levels immediately increased to $\geq 240 \mathrm{mg} / \mathrm{dL}$. LDL-C levels subsequently reduced again with evolocumab plus background LLT to an LDL-C of $107 \mathrm{mg} / \mathrm{dL}$. Responsiveness to evolocumab would be expected in a patient that has one wild-type $L D L R$ allele.

During lomitapide therapy, two assessments of CIMT were made. The first, at age 40 years while the patient was recently commenced on lomitapide $5 \mathrm{mg}$ /day, gave a result of $0.56 \mathrm{~mm}$. The second measure was made 26 months later when the patient was on lomitapide $30 \mathrm{mg} /$ day. This revealed CIMT of $0.57 \mathrm{~mm}$. Total plaque area had, however, regressed from 64.5 to 42.1 $\mathrm{mm}^{2}$ during the lomitapide treatment period.

\section{Patient 5}

Patient 5 is a woman with a homozygous p.Asp227Glu (defective) mutation in LDLR. The patient was diagnosed with $\mathrm{HoFH}$ at the age of 3 years. At age 39 years, the patient's interval mean LDL-C was $266.8 \mathrm{mg} / \mathrm{dL}$. She was receiving rosuvastatin $40 \mathrm{mg} /$ day, ezetimibe $10 \mathrm{mg} /$ day and colesevelam $375 \mathrm{mg} /$ day. LA was applied weekly and was very awkward for the patient's working life. In the same year, lomitapide was commenced at $5 \mathrm{mg}$ /day and escalated to $10 \mathrm{mg} /$ day 3 months later (Fig. 3). At this point, a mean CIMT reading was found to be $2.7 \mathrm{~mm}$. Lomitapide was escalated to $20 \mathrm{mg} /$ day, and LDL-C levels remained in the range $92-200 \mathrm{mg} / \mathrm{dL}$. LA intervals were gradually extended until lomitapide was escalated to $30 \mathrm{mg} /$ day, enabling cessation of LA with LDL-C levels as low as $46.4 \mathrm{mg} / \mathrm{dL}$ (nadir). Carotid ultrasound 9 months later, when the patient was on lomitapide $30 \mathrm{mg} /$ day but still undergoing monthly LA, revealed mean CIMT had 

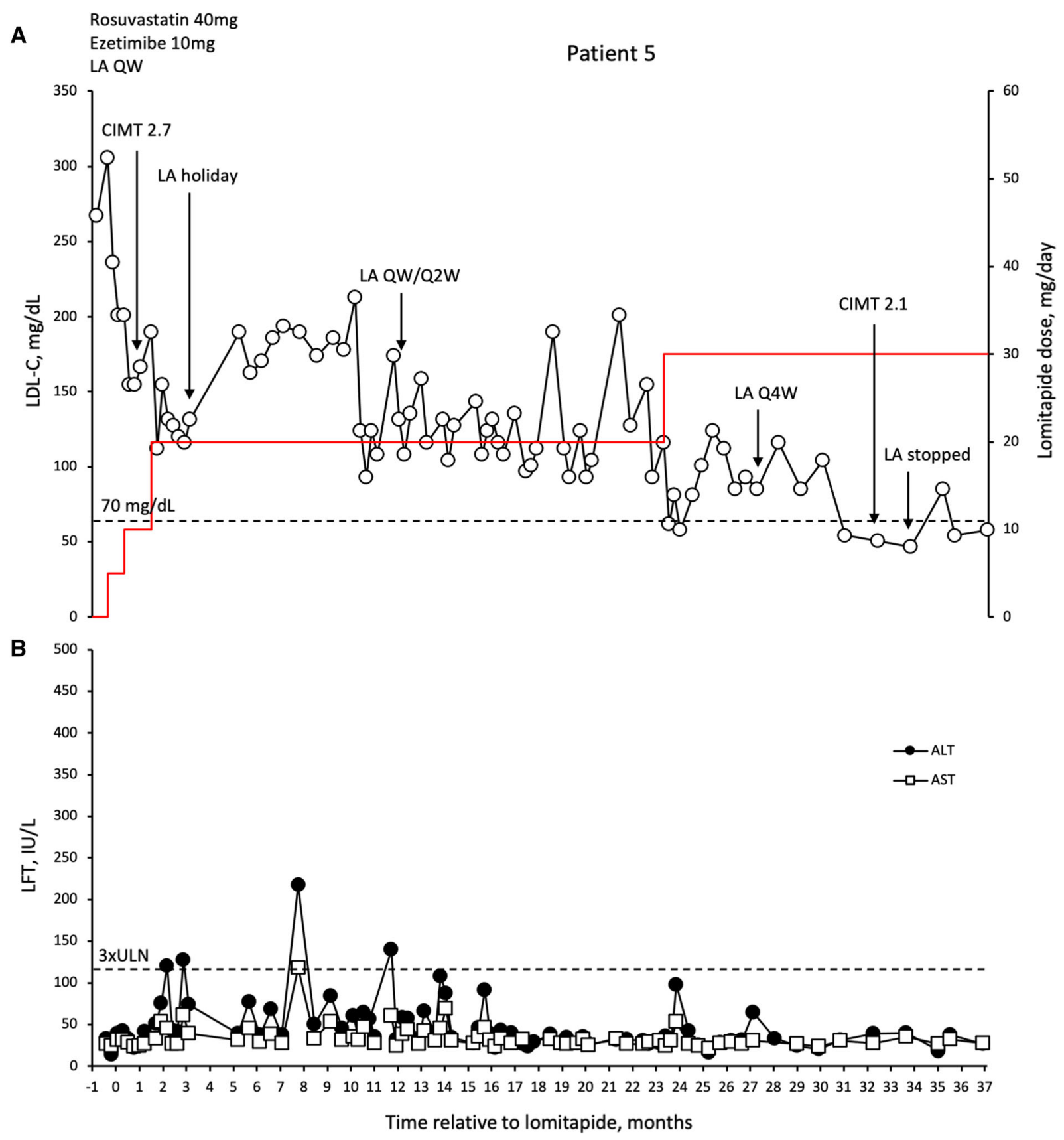

Fig. 3 A LDL-C levels, lomitapide dose (red line), CIMT, and B LFTs in Patient 5. ALT alanine aminotransferase, $A S T$ aspartate aminotransferase, CIMT carotid intimamedia thickness, $L A$ lipoprotein apheresis, $L D L-C$ low-

regressed to $2.1 \mathrm{~mm}$. LFTs increased temporarily but remained $<3$-times ULN and resolved without the need for specific intervention. density lipoprotein cholesterol, $L F T$ liver function test, $Q W$ once weekly, $Q 2 W$ once every 2 weeks, $Q 4 W$ once every 4 weeks, $U L N$ upper limit of normal

\section{Patient 6}

Patient 6 is a boy with a homozygous p.Glu228Ter (null/null) mutation in LDLR. At 
Table 1 Summary of CIMT measurements from baseline to follow-up in all six initial patients

\begin{tabular}{llllllll}
\hline Patient & $\begin{array}{l}\text { Baseline } \\
\text { CIMT, } \\
\text { mm }\end{array}$ & $\begin{array}{l}\text { Last } \\
\text { CIMT, } \\
\text { mm }\end{array}$ & $\begin{array}{l}\text { Interval } \\
\text { between } \\
\text { CIMT, years }\end{array}$ & $\begin{array}{l}\text { Interval between } \\
\text { lomitapide start and } \\
\text { last CIMT, years }\end{array}$ & $\begin{array}{l}\text { Change in } \\
\text { CIMT, } \\
\text { mm }\end{array}$ & $\begin{array}{l}\text { Net } \\
\text { response }\end{array}$ & $\begin{array}{l}\text { Measurement } \\
\text { site }\end{array}$ \\
\hline 1 & 1.18 & 1.11 & 8 & 7 & -0.08 & Regression & RCCA \\
2 & 0.82 & 0.65 & 2 & 4 & -0.17 & Regression & RCCA, LCCA \\
3 & 0.76 & 0.80 & 10 & 9 & 0.04 & Stable & RCCA, LCCA \\
4 & 0.56 & 0.57 & 2 & 2 & 0.01 & Stable & RCCA, LCCA \\
5 & 2.65 & 2.05 & 3 & 2 & -0.60 & Regression & RCCA, LCCA \\
6 & 1.81 & 1.20 & 5 & 2 & -0.61 & Regression & RCCA, LCCA \\
Mean & 1.30 & 1.06 & 4.9 & & -0.23 & & \\
SD & 0.80 & 0.54 & 3.3 & & 0.30 & & \\
Median & 1.00 & 0.95 & 3.9 & & -0.12 & & \\
Min & 0.56 & 0.57 & 2.0 & & -0.61 & & \\
Max & 2.65 & 2.05 & 9.9 & & 0.04 & & \\
\hline
\end{tabular}

CIMT carotid intima-media thickness, LCCA left common carotid artery, Max maximum, Min minimum, RCCA right common carotid artery, $S D$ standard deviation

the age of 8 years, the patient had mean CIMT $1.81 \mathrm{~mm}$ against a background of untreated LDL-C levels of $658 \mathrm{mg} / \mathrm{dL}$. The patient was commenced on rosuvastatin $20 \mathrm{mg} /$ day and ezetimibe $10 \mathrm{mg} /$ day. LA was commenced once every 2 weeks later the same year, and CIMT reached $1.39 \mathrm{~mm}$ a year later. LDL-C levels decreased to $450 \mathrm{mg} / \mathrm{dL}$ over the course of the next 3 years (Supplemental Fig. 4), and some regression of CIMT was evident (i.e. $1.56 \mathrm{~mm}$ at 26 months after first CIMT). Forty months after the first CIMT, a repeat CIMT was $1.29 \mathrm{~mm}$ and lomitapide was commenced at $5 \mathrm{mg} /$ day and escalated to $40 \mathrm{mg} /$ day in a stepwise fashion. Ten months after starting lomitapide, adjustments were made to the patient's LA schedule such that there was less burden of LA, and he remains on monthly LA. During this period, serial CIMT measures were made, which showed sustained regression of CIMT down to $1.20 \mathrm{~mm}$. LDL-C has been maintained in the range $100-220 \mathrm{mg} / \mathrm{dL}$ (nadir $98.0 \mathrm{mg} / \mathrm{dL}$ ) with no relevant changes in LFTs.

\section{Summary of CIMT data from six core patients}

A summary of CIMT measures for all six patients is given in Table 1. There is a general trend towards stabilisation or regression of CIMT in the six core cases (Supplemental Fig. 5).

Differences in ultrasound techniques and analysis protocols between patients means that information on plaque area is not available for most patients. Where these data are available (Patients 2 and 4), modest regression or stabilisation in CIMT was accompanied by clinically significant regression of plaque area (Table 2).

\section{Additional data from Greece}

The Greek cohort included five male and three female patients. All patients had markedly elevated LDL-C levels prior to initiation of lomitapide (mean $435.1 \pm 94.6 \mathrm{mg} / \mathrm{dL}$; range $345-600 \mathrm{mg} / \mathrm{dL}$ ), despite use of maximal daily dose of rosuvastatin $(40 \mathrm{mg} /$ day plus ezetimibe $10 \mathrm{mg} /$ day). Mean reductions in LDL-C of $69 \pm 11 \%$ (nadir) were achieved with a mean 
dose of lomitapide $31 \pm 10 \mathrm{mg} /$ day. Only one patient (Patient 5) experienced elevations in AST and ALT $>3$ times ULN. Mean (left and right common carotid arteries) CIMT prior to lomitapide therapy was in the range 0.6-1.1 mm, and CIMT values at follow-up demonstrated general stability of CIMT (change in CIMT $0.04 \pm 0.24 \mathrm{~mm}$, with $50 \%$ of the patients showing some evidence of CIMT regression (Table 3 ).

Mutation classifications for all patients are shown in Supplemental Table 1.

\section{DISCUSSION}

This case series shows that CIMT regresses or stabilises in the majority of $\mathrm{HoFH}$ patients treated with lomitapide when LDL-C is controlled. All the patients (including the patient with severe $\mathrm{HeFH}$ ) in this case series had extremely elevated levels of LDL-C characteristic of $\mathrm{HoFH}$, which were not adequately controlled by conventional lipid-lowering therapy, including LA in many cases.

In all patients, lomitapide resulted in marked decreases in LDL-C levels. At nadir, LDL-C was reduced by $76.5-93.9 \%$. Overall, eight patients (six from the original cohort, and two from Greece) were able to achieve LDL-C levels $<100 \mathrm{mg} / \mathrm{dL}$, and three patients were able to achieve LDL-C $<70 \mathrm{mg} / \mathrm{dL}$.

In the present series, patients were all treated in a real-world setting. Previous experience of case series outside of clinical trials have shown that lomitapide reduces LDL-C levels to the same extent or greater as that seen in phase 3 clinical trials, but with lower doses [14-18]. This likely occurs because the phase 3 trial used a forced titration study design whereby lomitapide doses were escalated until the patient was not able to tolerate the adverse events [9]. In the real world, lomitapide tends to be dosed to reduce LDL-C levels or reduce LA burden, so doses are often lower, titration is slower and the adverse event profile is less severe. In the current series, elevations in LFTs were not uncommon, but all resolved with minimal intervention required.
Table 2 Summary carotid artery plaque data in two patients

\begin{tabular}{|c|c|c|}
\hline \multirow[t]{2}{*}{ Parameter } & \multicolumn{2}{|l|}{ Patient } \\
\hline & 2 & 4 \\
\hline Change in mean CIMT, $\mathrm{mm}$ & -0.17 & 0.01 \\
\hline Change in total plaque area, $\mathrm{mm}^{2}$ & -26.6 & -22.39 \\
\hline Change in mean total thickness, $\mathrm{mm}$ & 0.01 & -0.27 \\
\hline Change in total area, $\mathrm{mm}^{2}$ & -30 & -22.19 \\
\hline Change in vascular age, years & 0 & -10 \\
\hline
\end{tabular}

CIMT carotid intima-media thickness

This case series shows for the first time that the LDL-C lowering brought about by lomitapide also translates into CIMT regression or arrest of progression in patients with HoFH. Until now, the only data indicating that lomitapide might have any effect on long-term cardiovascular and survival outcomes are derived from a modelling analysis that applied lomitapide-associated reductions in LDL-C levels to historical data from a South African cohort in which survival data were available according to LDL-C level. This analysis suggested that additional LDL-C lowering by lomitapide may increase life expectancy in patients with $\mathrm{HoFH}$ by 11.2 years and increase time to first MACE by 5.7 years (6.7 years with lifetime exposure to lomitapide) [19].

The case series we have presented has several limitations. There is no control group that did not take lomitapide, and as this is an ultra-rare disease, the sample size is small. One of the patients (Patient 4) did not have HoFH, rather, he had a severe heterozygous FH phenotype, but this does not detract from the value of understanding the $\mathrm{CV}$ outcomes for lomitapide in a patient with high LDL-C levels. In addition, we do not have plaque area data in all patients. Nevertheless, the data presented here do enable us to see, for the first time, the effect of lomitapide on vascular health as measured by changes in CIMT.

CIMT is an established surrogate marker for progression of atherosclerotic disease $[12,20-22]$, and recently was recommended by 


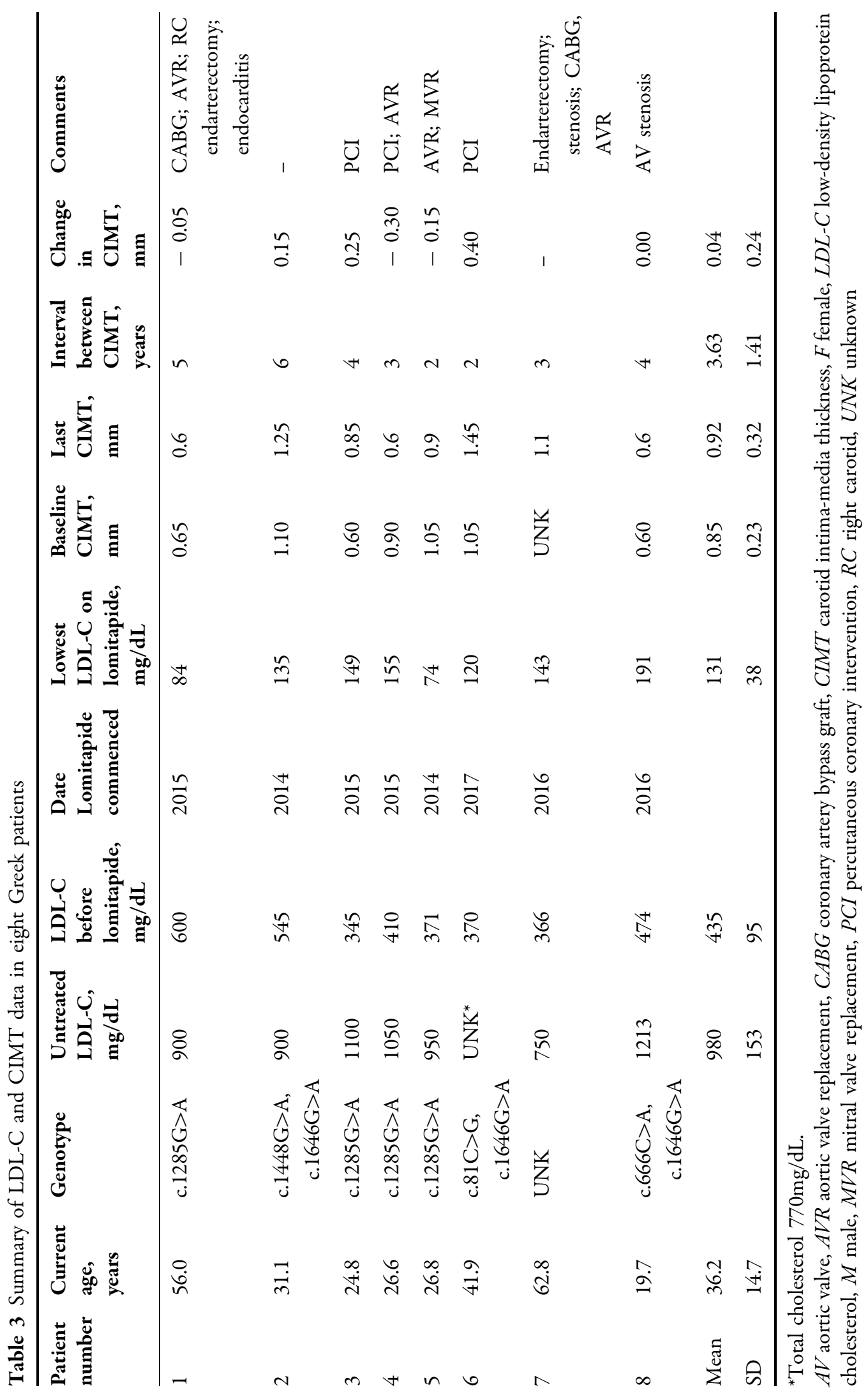


a 2019 ESC/EAS guidelines committee for assessment of arterial plaque burden [23]. Two large studies-The Rotterdam Study [24] and the Atherosclerosis in Communities (ARIC) Study [25] - are the pivotal studies that established CIMT as a marker for progressive atherosclerotic disease. In the Rotterdam study of 8000 persons $>55$ years of age, ultrasound studies produced solid evidence that CIMT measures can be used as an indicator of atherosclerosis [24]. In the ARIC Study of 15,800 adults, high-resolution B-mode ultrasound was able to assess and delineate all stages of atherosclerosis [25]. Subsequent work has built on these findings, and CIMT is now routinely used as an accurate and inexpensive means of assessing cardiovascular progression or regression in pharmaceutical or population cohort studies [12, 20-22].

In the patients described in the current case series, regression of CIMT was seen in $50 \%$ of patients, while in the remaining patients, CIMT did not increase further, often over lengthy observation periods. This lack of regression should, however, not be interpreted as a negative finding, and is similar to the lack of regression seen in the ENHANCE study of patients with heterozygous $\mathrm{FH}$ treated either with simvastatin $80 \mathrm{mg} /$ day or simvastatin $80 \mathrm{mg}$ /day plus ezetimibe $10 \mathrm{mg} /$ day. In the ENHANCE trial [26], most participants were enrolled from lipid clinics and had been treated aggressively prior to enrolment. Baseline CIMT in ENHANCE was $0.69 \mathrm{~mm}$, much lower than the value of $0.93 \mathrm{~mm}$ found in the earlier ASAP trial, which was able to demonstrate regression, and in which most patients had not been exposed previously to aggressive lipid-lowering therapy [27]. These and other trials show that, if the CIMT is not elevated at baseline, it is not possible to demonstrate CIMT regression. However, lack of progression is very encouraging given the natural tendency for atherosclerotic progression, particularly among $\mathrm{HoFH}$ patients [28]. In the present case series, the greatest regression was seen in the patient with the highest baseline CIMT. Conceptually, lipidrich plaques are more likely to exhibit significant regression compared to plaques with significant fibrosis and calcification.
In addition to CIMT, additional imaging techniques are available that could assist in the assessment of atherosclerotic risk in HoFH. In Patients 2 and 4, additional imaging was available that enabled assessment of total plaque area (Table 2). In both patients, while the alterations in CIMT are modest or absent, the total plaque areas regressed by a considerable amount. This underscores the value of more detailed imaging techniques to detect changes in atherosclerotic burden [29]. The present, case-based evaluation of CIMT in patients with $\mathrm{HoFH}$ is limited by non-standard protocols and lack of between-group comparisons.

Atherosclerotic burden in $\mathrm{HoFH}$ can be evaluated by multiple means, including coronary artery calcium scoring and MRI; however, ultrasound-based techniques are non-invasive, relatively cost-effective and are also not associated with exposure to ionizing radiation. Computed tomography coronary angiography, angiography and myocardial scintigraphy are most useful to evaluate vascular anatomy or myocardial perfusion [30, 31]. Although guidelines recommend regular imaging in patients with HoFH [15], the goal of such imaging is to detect coronary artery or aortic valve stenosis, rather than quantifying atherosclerotic burden.

In conclusion lomitapide reduces LDL-C levels in patients with HoFH and very severe LDL-C phenotypes, and also results in stabilisation and/or regression of CIMT, an established marker of ASCVD.

\section{ACKNOWLEDGEMENTS}

Funding. Patients in this case series were treated in the normal course of their care, and no funding was supplied for this. Some patients were enrolled in clinical trials of lomitapide, which were funded by Amryt Pharmaceuticals DAC. The Journal's Rapid Service and Open Access fees were also funded by Amryt.

Medical Writing and/or Editorial Assistance. The authors thank Mr Adam D McIntyre for assistance with variant nomenclature and classification, and Dr Nigel C Eastmond of 
Eastmond Medicomm Ltd for editorial assistance with the collation of data and the preparation of this manuscript. Editorial support was funded by Amryt Pharmaceuticals DAC.

Authorship. All named authors meet the International Committee of Medical Journal Editors (ICMJE) criteria for authorship for this article, take responsibility for the integrity of the work, and have given their approval for this version to be published.

Author Contributions. All authors contributed to the treatment of the patients and the design of their treatment protocols. Material preparation, data collection and analysis were performed by all authors. The first draft of the manuscript was written by Dirk Blom and all authors commented on the manuscript. All authors read and approved the final manuscript

Prior Presentation. An abstract and poster including some of the data from this manuscript were presented at the National Lipid Association Scientific Sessions, September 24-26 2021 in Orlando, Florida, USA.

Disclosures. Dirk Blom has received honoraria and consulting fees from Amryt, Aegerion, Amgen and Sanofi Aventis. Dirk Blom chairs the steering committee of the LOWER observational study of lomitapide sponsored by Amryt. Daniel Gaudet has received research grant support from Acasti, Akcea, Aegerion, Amgen, Amryt, AstraZeneca, Boehringer Ingelheim, Canadian Cardiovascular Research Network, Cerenis, Dalcor Pharma, Esperion, Gemphire, GlaxoSmithKline, HDL Therapeutics, Ionis Pharmaceuticals, Ironwood, Lilly, Kowa, Novartis, Pfizer, Regeneron, Sanofi, UniQure, and has served as a consultant for Akcea, Aegerion, Amgen, HDL Therapeutics, Ionis Pharmaceuticals, Novartis, Regeneron and Sanofi. Robert A Hegele reports honoraria and consulting fees from Acasti, Akcea/Ionis, Amgen, Amryt, Arrowhead, HLS Therapeutics, Novartis, Pfizer, Regeneron and Sanofi. Dharmesh Patel has received honoraria and consulting fees from Amgen and is a principal investigator on the Novartis-sponsored Heritage and Horizon trials in patients with elevated Lp(a). Jaimini Cegla has received honoraria and research grants from Amryt, Amgen, Sanofi and Novartis. Genovefa Kolovou participated in research and consulting activities sponsored by healthcare companies, including Amgen, Amryt, MSD, Sanofi, and Servier. Luis Masana Marin has received honoraria for lectures and advisory work from Amgen, Amryt, Amarin, Mylan, Servier, Novartis, Sanofi.

Compliance and Ethics Guidelines. This case series was conducted as a retrospective study of normal patient care and is not subject to Institutional Review Board approval. The study was performed in accordance with the Helsinki Declaration of 1964, and its later amendments. All of the patients provided written consent for their details to be published in the current case series. In the instances where patients died, consent was secured from the patients' estates and/or living relatives. The authors thank the patients and families.

Data Availability. The full data set is not provided as it contains identifying information, but can be secured on reasonable request from the corresponding author.

Open Access. This article is licensed under a Creative Commons Attribution-NonCommercial 4.0 International License, which permits any non-commercial use, sharing, adaptation, distribution and reproduction in any medium or format, as long as you give appropriate credit to the original author(s) and the source, provide a link to the Creative Commons licence, and indicate if changes were made. The images or other third party material in this article are included in the article's Creative Commons licence, unless indicated otherwise in a credit line to the material. If material is not included in the article's Creative Commons licence and your intended use is not permitted by statutory regulation or exceeds the permitted use, you will need to obtain permission directly from the copyright holder. To view a copy of this licence, visit http://creativecommons.org/licenses/by$\mathrm{nc} / 4.0 /$. 


\section{REFERENCES}

1. Cuchel M, Bruckert E, Ginsberg HN, Raal FJ, Santos $\mathrm{RD}$, Hegele RA et al. Homozygous familial hypercholesterolaemia: new insights and guidance for clinicians to improve detection and clinical management. A position paper from the consensus panel on familial hypercholesterolaemia of the european atherosclerosis society. Eur Heart J. 2014;35(32):2146-57. doi:https://doi.org/10.1093/ eurheartj/ehu274.

2. Hobbs HH, Brown MS, Goldstein JL. Molecular genetics of the LDL receptor gene in familial hypercholesterolemia. Hum Mutat. 1992;1(6): 445-66. 1380010602.

3. Robinson JG. Management of familial hypercholesterolemia: a review of the recommendations from the national lipid association expert panel on familial hypercholesterolemia. J Manag Care Pharm. 2013;19(2):139-49.

4. Nordestgaard BG, Chapman MJ, Humphries SE, Ginsberg HN, Masana L, Descamps OS, et al. Familial hypercholesterolaemia is underdiagnosed and undertreated in the general population: guidance for clinicians to prevent coronary heart disease: consensus statement of the European atherosclerosis society. Eur Heart J. 2013;34(45): 3478-90. https://doi.org/10.1093/eurheartj/eht273.

5. Santos RD, Stein EA, Hovingh GK, Blom DJ, Soran $H$, Watts GF, et al. Long-term evolocumab in patients with familial hypercholesterolemia. J Am Coll Cardiol. 2020;75(6):565-74. https://doi.org/10. 1016/j.jacc.2019.12.020.

6. Aegerion Pharmaceuticals Inc. Juxtapid prescribing information. 2013.

7. Amryt Pharmaceuticals DAC. Lojuxta summary of product characteristics. 2017.

8. Cuchel M, Bloedon LT, Szapary PO, Kolansky DM, Wolfe ML, Sarkis A, et al. Inhibition of microsomal triglyceride transfer protein in familial hypercholesterolemia. N Engl J Med. 2007;356(2):148-56. https://doi.org/10.1056/NEJMoa061189.

9. Cuchel M, Meagher EA, du Toit TH, Blom DJ, Marais AD, Hegele RA, et al. Efficacy and safety of a microsomal triglyceride transfer protein inhibitor in patients with homozygous familial hypercholesterolaemia: a single-arm, open-label, phase 3 study. Lancet. 2013;381(9860):40-6. https://doi. org/10.1016/S0140-6736(12)61731-0.

10. Blom DJ, Cuchel M, Ager M, Phillips H. Target achievement and cardiovascular event rates with
Lomitapide in homozygous Familial Hypercholesterolaemia. Orphanet J Rare Dis. 2018;13(1):96. https://doi.org/10.1186/s13023-018-0841-3.

11. Bauer M, Caviezel S, Teynor A, Erbel R, Mahabadi AA, Schmidt-Trucksass A. Carotid intima-media thickness as a biomarker of subclinical atherosclerosis. Swiss Med Wkly. 2012;142: w13705. https:// doi.org/10.4414/smw.2012.13705.

12. de Groot E, Hovingh GK, Wiegman A, Duriez P, Smit AJ, Fruchart JC et al. Measurement of arterial wall thickness as a surrogate marker for atherosclerosis. Circulation. 2004;109(23 Suppl 1): Iii33-8. https://doi.org/10.1161/01.CIR. 0000131516.65699.ba.

13. Willeit P, Tschiderer L, Allara E, Reuber K, Seekircher L, Gao L, et al. Carotid intima-media thickness progression as surrogate marker for cardiovascular risk: meta-analysis of 119 clinical trials involving 100667 patients. Circulation. 2020;142(7):621-42. https://doi.org/10.1161/ CIRCULATIONAHA.120.046361.

14. Ben-Omran T, Masana L, Kolovou G, Ariceta G, Novoa FJ, Lund AM, et al. Real-world outcomes with lomitapide use in paediatric patients with homozygous familial hypercholesterolaemia. Adv Ther. 2019;36(7):1786-811. https://doi.org/10. 1007/s12325-019-00985-8.

15. Blom DJ, Kastelein JJ, Larrey D, Makris L, Schwamlein C, Phillips $\mathrm{H}$, et al. Lomitapide observational worldwide evaluation registry (LOWER): oneyear data. Circulation. 2015;132:A10818.

16. D'Erasmo L, Cefalu AB, Noto D, Giammanco A, Averna M, Pintus P, et al. Efficacy of lomitapide in the treatment of familial homozygous hypercholesterolemia: results of a real-world clinical experience in Italy. Adv Ther. 2017;34(5):1200-10. https://doi.org/10.1007/s12325-017-0531-x.

17. Roeters van Lennep J, Averna M, Alonso R. Treating homozygous familial hypercholesterolemia in a real-world setting: experiences with lomitapide. J Clin Lipidol. 2015;9(4):607-17. https://doi.org/10. 1016/j.jacl.2015.05.001.

18. Stefanutti C, Morozzi C, Di Giacomo S, Sovrano B, Mesce D, Grossi A. Management of homozygous familial hypercholesterolemia in real-world clinical practice: a report of 7 Italian patients treated in Rome with lomitapide and lipoprotein apheresis. J Clin Lipidol. 2016;10(4):782-9. https://doi.org/10. 1016/j.jacl.2016.02.009.

19. Leipold R, Raal F, Ishak J, Hovingh K, Phillips H. The effect of lomitapide on cardiovascular outcome measures in homozygous familial hypercholesterolemia: a modelling analysis. Eur J Prev Cardiol. 
2017;24(17):1843-50. 2047487317730473.

https://doi.org/10.1177/

20. Bots ML. Carotid intima-media thickness as a surrogate marker for cardiovascular disease in intervention studies. Curr Med Res Opin. 2006;22(11): 2181-90. $030079906 \times 148472$. https://doi.org/10.1185/

21. Bots ML, Grobbee DE. Intima media thickness as a surrogate marker for generalised atherosclerosis. Cardiovasc Drugs Ther. 2002;16(4):341-51. https:// doi.org/10.1023/a:1021738111273.

22. Fitch KV, Stavrou E, Looby SE, Hemphill L, Jaff MR, Grinspoon SK. Associations of cardiovascular risk factors with two surrogate markers of subclinical atherosclerosis: endothelial function and carotid intima media thickness. Atherosclerosis. 2011;217(2):437-40. https://doi.org/10.1016/j. atherosclerosis.2011.04.009.

23. Mach F, Baigent C, Catapano AL, Koskinas KC, Casula M, Badimon L, et al. 2019 ESC/EAS Guidelines for the management of dyslipidaemias: lipid modification to reduce cardiovascular risk. Eur Heart J. 2020;41(1):111-88. https://doi.org/10. 1093/eurheartj/ehz455.

24. Bots ML, Hoes AW, Koudstaal PJ, Hofman A, Grobbee DE. Common carotid intima-media thickness and risk of stroke and myocardial infarction: the Rotterdam study. Circulation. 1997;96(5): 1432-7. https://doi.org/10.1161/01.cir.96.5.1432.

25. Chambless LE, Heiss G, Folsom AR, Rosamond W, Szklo M, Sharrett AR, et al. Association of coronary heart disease incidence with carotid arterial wall thickness and major risk factors: the atherosclerosis risk in communities (ARIC) Study, 1987-1993. Am J
Epidemiol. 1997;146(6):483-94. https://doi.org/10. 1093/oxfordjournals.aje.a009302.

26. Kastelein JJ, Akdim F, Stroes ES, Zwinderman AH, Bots ML, Stalenhoef AF, et al. Simvastatin with or without ezetimibe in familial hypercholesterolemia. N Engl J Med. 2008;358(14):1431-43. https://doi.org/10.1056/NEJMoa0800742.

27. Smilde TJ, van Wissen S, Wollersheim H, Trip MD, Kastelein JJ, Stalenhoef AF. Effect of aggressive versus conventional lipid lowering on atherosclerosis progression in familial hypercholesterolaemia (ASAP): a prospective, randomised, double-blind trial. Lancet. 2001;357(9256):577-81. https://doi. org/10.1016/s0140-6736(00)04053-8.

28. Stein EA. After ENHANCE: is more LDL cholesterol lowering even better? Clin Chem. 2008;54(6): 940-2. https://doi.org/10.1373/clinchem.2008. 104893.

29. Al-Shali K, House AA, Hanley AJ, Khan HM, Harris SB, Mamakeesick $M$, et al. Differences between carotid wall morphological phenotypes measured by ultrasound in one, two and three dimensions. Atherosclerosis. 2005;178(2):319-25. https://doi. org/10.1016/j.atherosclerosis.2004.08.016.

30. Cremer P, Hachamovitch R, Tamarappoo B. Clinical decision making with myocardial perfusion imaging in patients with known or suspected coronary artery disease. Semin Nucl Med. 2014;44(4):320-9. https://doi.org/10.1053/j.semnuclmed.2014.04. 006.

31. Rahsepar AA, Arbab-Zadeh A. Cardiac CT vs. Stress Testing in Patients with Suspected Coronary Artery Disease: Review and Expert Recommendations. Curr Cardiovasc Imaging Rep. 2015;8(8). doi: https://doi.org/10.1007/s12410-015-9344-y. 